\title{
Schnorr triviality and its equivalent notions
}

\author{
Kenshi Miyabe*
}

March 23, 2013

\begin{abstract}
We give some new characterizations of Schnorr triviality. In concrete terms, we introduce a reducibility related to decidable prefix-free machines and show the equivalence with Schnorr reducibility. We also give a uniform-Schnorr-randomness version of the equivalence of LR-reducibility and LK-reducibility. Finally we prove a base-type characterization of Schnorr triviality.
\end{abstract}

Keywords: algorithmic randomness, Schnorr randomness, Schnorr triviality, uniform relativization, lowness for uniform Schnorr randomness

\section{Introduction}

The theory of algorithmic randomness [21,9] gives the definition of that a set is random. At the same time, the theory also studies a set that is "far from random" or "randomness theoretically weak".

Martin-Löf randomness [16] is the randomness notion that has been studied the most. One formulation of "far from ML-random" is $K$-triviality and this notion has many characterizations. For instance, the following equivalence for a set $A[20,12]$ :

(i) $A$ is $K$-trivial, that is, $K(A\lceil n) \leq K(n)+O(1)$,

(ii) $A$ is low for Martin-Löf randomness, that is, each Martin-Löf random set is Martin-Löf random relative to $A$,

(iii) $A$ is low for prefix-free Kolmogorov complexity, that is, $K(\sigma) \leq K^{A}(\sigma)+$ $O(1)$,

(iv) $A$ is a base for Martin-Löf randomness, that is, $A$ is Turing reducible to a Martin-Löf random set relative to $A$.

\footnotetext{
${ }^{*}$ Research Institute for Mathematical Sciences, Kyoto University, kmiyabe@kurims.kyotou.ac.jp
} 
Note that (i) means that $A$ is far from random by the Levin-Schnorr theorem. The equivalence between (ii) and (iii) was strengthened to the equivalence between LR-reducibility and LK-reducibility [14].

Schnorr randomness [22] is another important randomness notion. Similarly, the lowness notions for Schnorr randomness have been studied [7, 8, 15, 6]. Unlike the case of Martin-Löf randomness, lowness for Schnorr randomness is not equivalent to Schnorr triviality, which is a Schnorr-randomness version of $K$-triviality [8].

Franklin and Stephan [10] and Miyabe [18] claimed that truth-table reducibility is more suitable than Turing reducibility when studying a notion of relativized Schnorr randomness and proposed truth-table Schnorr randomness (tt-Schnorr randomness) and showed the equivalence of the following:

(i) Schnorr triviality,

(ii) computable tt-traceability,

(iii) lowness for tt-Schnorr randomness,

(iv) lowness for truth-table reducible measure machines.

Franklin and Stephan [10] also showed the equivalence to a kind of being a base for Schnorr randomness that uses new reducibility $\leq_{\text {snr. }}$. We would like to have a notion of being a base for Schnorr randomness that uses truth-table reducibility.

Miyabe [18] claimed that truth-table Schnorr randomness is the proper relativization of Schnorr randomness that is different from the usual relativization. Subsequently, Miyabe and Rute [19] introduced uniform relativization. The uniform relativization of Schnorr randomness is called uniform Schnorr randomness, which is equivalent to tt-Schnorr randomness but has a slightly different formulation.

In this paper we give some new characterizations of Schnorr triviality. In Section 3 we give a characterization of Schnorr reducibility via decidable prefixfree machines. Schnorr reducibility is defined via computable measure machines, with which Schnorr randomness has a characterization. Since we have a characterization of Schnorr randomness via decidable prefix-free machines, such a characterization should be studied. In Section 4 we study a uniformSchnorr-randomness version of the equivalence between LK-reducibility and LRreducibility. We also give characterizations via open covers. In Section 5 we give a base-type characterization of Schnorr triviality. More precisely, we will show the equivalence among the following:

(i) Schnorr triviality,

(ii) being a base for uniform Schnorr tests,

(iii) being a base for uniformly computable martingales.

The main tool is a uniformly-computable-martingale version of the Kucěra-Gács Theorem. It should be noted that Franklin, Stephan and Yu [11] studied a base for Schnorr randomness, which is a notion different from the one considered in our study. 


\section{Preliminary}

We refer to the books $[21,9]$ for the notions in algorithmic randomness. By $2^{<\omega}$, we denote the set of binary strings. Cantor space $2^{\omega}$ is the set of infinite binary sequences equipped the canonical topology. By $\preceq$, we denote the prefix relation. For $\sigma \in 2^{<\omega}$, we denote by $\llbracket \sigma \rrbracket$ the set of all extensions of $\sigma$ in $2^{\omega}$, that is, $\llbracket \sigma \rrbracket=\left\{X: \sigma \prec X \in 2^{\omega}\right\}$. The uniform measure on $2^{\omega}$ is denoted by $\mu$. We identify a set $X \subseteq \mathbb{N}$ with the binary sequence $X$ by $n \in X \Longleftrightarrow X(n)=1$ where $X(n)$ is the $n$-th bit of $X$.

\subsection{Schnorr randomness}

The following are basic definitions and results on Schnorr randomness.

A Schnorr test is a sequence $\left\{U_{n}\right\}$ of uniformly c.e. open sets such that $\mu\left(U_{n}\right) \leq 2^{-n}$ for all $n$ and the measure $\mu\left(U_{n}\right)$ is uniformly computable in $n$. A set $X \in 2^{\omega}$ passes a Schnorr test $\left\{U_{n}\right\}$ if $x \notin \bigcap_{n} U_{n}$. A set is called Schnorr random if it passes all Schnorr tests.

A machine is a partial computable function from $2^{<\omega}$ to $2^{<\omega}$. For a machine $M$, Kolmogorov complexity $K$ of $\tau \in 2^{<\omega}$ with respect to $M$ is defined by $K_{M}(\tau)=\min \{|\sigma|: M(\sigma)=\tau\}$. A set of strings is prefix-free if, for two disjoint strings in the set, one is not a prefix of the other. A machine is called prefix-free if its domain is prefix-free. A computable measure machine is a prefixfree machine $M$ such that $\mu(\llbracket \operatorname{dom}(M) \rrbracket)=\sum_{\sigma \in \operatorname{dom}(M)} 2^{-|\sigma|}$ is computable. A set $X \in 2^{\omega}$ is Schnorr random iff $K_{M}(X\lceil n) \geq n-O(1)$ for all computable measure machines $M$ [7].

A machine $M$ is called decidable if $\operatorname{dom}(M)$ is computable. An order is an unbounded nondecreasing function from $\mathbb{N}$ to $\mathbb{N}$.

Theorem 2.1 (Bienvenu and Merkle [1]). A set $X$ is Schnorr random iff for all decidable prefix-free machines $M$ and all computable orders $g$, we have $K_{M}(X \uparrow$ $n) \geq n-g(n)-O(1)$.

A martingale is a function $d: 2^{<\omega} \rightarrow \mathbb{R}^{+}$such that $2 d(\sigma)=d(\sigma 0)+d(\sigma 1)$ for all $\sigma \in 2^{<\omega}$. A set $X$ is Schnorr random iff $d(X\lceil n) \leq g(n)$ for all but finite $n$ for each computable martingale $d$ and each computable order $g$.

\subsection{Uniform Schnorr and computable randomness}

When relativizing a randomness notion, we need to be careful. One criterion of naturalness of a relativization is whether van Lambalgen's theorem holds or not $[18,5]$. Miyabe [18] and Miyabe and Rute [19] showed that van Lambalgen theorem holds for uniform Schnorr randomness. The following definitions and results in this subsection are essentially from Miyabe [18] and Miyabe and Rute [19] although the formulations are slightly different.

Definition 2.2. A uniform Schnorr test is a computable functions $f: 2^{\omega} \times \omega \rightarrow$ $\tau$ such that the function $\langle X, n\rangle \mapsto \mu(f(X, n))$ is computable and $\mu(f(X, n)) \leq$ 
$2^{-n}$ for all $X \in 2^{\omega}$ and $n \in \omega$. We say that a sequence $\left\{U_{n}^{A}\right\}$ is a Schnorr test uniformly relative to $A$ if $U_{n}^{A}=f(A, n)$ for all $n$ for a uniform Schnorr test $f$. $A$ set $B$ is called Schnorr random uniformly relative to $A$ if $B \notin \bigcap_{n} U_{n}^{A}$ for a Schnorr test $\left\{U_{n}^{A}\right\}$ uniformly relative to $A$.

Here $\tau$ is the set of open sets on $2^{\omega}$. We refer to $[24,3,4,25]$ for computability from $2^{\omega}$ to $\tau$, from $2^{\omega}$ to $\mathbb{R}$ and so on. Miyabe and Rute [19] showed that uniform Schnorr randomness is equivalent to tt-Schnorr randomness studied in $[10,18]$.

Uniform Schnorr randomness has characterizations via complexity and martingales.

Definition 2.3. An oracle prefix-free machine $M$ is a uniformly computable measure machine if the function $X \mapsto \mu\left(\llbracket \operatorname{dom}\left(M^{X}\right) \rrbracket\right)$ is computable.

A uniformly computable measure machine is called a truth-table reducible measure machine in [18].

Theorem 2.4 ([18]). A set $B$ is Schnorr random uniformly relative to $A$ iff $K_{M^{A}}(B \nmid n)>n-O(1)$ for all uniformly computable measure machine $M$.

Definition 2.5. A uniform martingale test is a computable map $d: 2^{\omega} \times 2^{<\omega} \rightarrow$ $\mathbb{R}^{+}$such that $d^{Z}=d(Z, \cdot)$ is a martingale for each $Z \in 2^{\omega}$. In this case, we say that such a martingale $d^{A}$ is computable uniformly relative to $A$.

See also the definition in Franklin and Stephan [10].

Theorem 2.6 ([10, 18]). A set $B$ is Schnorr random uniformly relative to $A$ iff $d^{A}\left(B\lceil n) \leq h(n)\right.$ for almost all $n$ for each martingale $d^{A}$ computable uniformly relative to $A$ and each computable order $h$.

\subsection{Lowness notions for uniform Schnorr randomness}

Here we review lowness notions for uniform Schnorr randomness. Notice that we modify the terminology according to the change of the name of uniform Schnorr randomness.

Definition 2.7 (Downey et al. [7]). A set $A$ is Schnorr reducible to a set $B$ (denoted by $A \leq_{\mathrm{Sch}} B$ ) if for each computable measure machine $M$ there is a computable measure machine $N$ such that $K_{N}\left(A\lceil n) \leq K_{M}(B\lceil n)+O(1)\right.$. $A$ set $A$ is Schnorr trivial if $A \leq_{\text {Sch }} \emptyset$.

Definition 2.8. A trace is a sequence $\left\{T_{n}\right\}$ of sets. A trace $\left\{T_{n}\right\}$ is a trace for a total function $f$ if $f(n) \in T_{n}$ for all $n$. We say a trace $\left\{T_{n}\right\}$ traces a function $f$ if $\left\{T_{n}\right\}$ is a trace for $f$. A set $A$ is computably tt-traceable via a computable order $h$ if all functions $f \leq_{\mathrm{tt}} A$ are traced by an $h$-bounded c.e. trace $\left\{T_{n}\right\}$. We say that a set $A$ is computably tt-traceable if it is computable tt-traceable for some computable order $h$.

Note that a set is computably tt-traceable iff it is computably tt-traceable for all computable orders. For details, see [23], [21, Theorem 8.2.3] and [13]. 
Definition 2.9. A set $A$ is low for uniform Schnorr randomness if every Schnorr random set is Schnorr random uniformly relative to $A$. A set $A$ is low for uniformly computable measure machines if for each uniformly computable measure machine $M$ there is a computable measure machine $N$ such that $K_{N}(\sigma) \leq$ $K_{M^{A}}(\sigma)+O(1)$.

Theorem 2.10 (Franklin and Stephan [10, Theorem 3.2], Miyabe [18, Theorem 7.3]). The following are equivalent for a set $A \in 2^{\omega}$ :

(i) A is Schnorr trivial,

(ii) A is computably tt-traceable,

(iii) A is low for uniform Schnorr randomness,

(iv) A is low for uniformly computable measure machines.

We also use the following result.

Definition 2.11 (Hölzl and Merkle [13]). A set $A$ is totally i.o. complex if there is a computable function $h$ such that for all total machines $M$ there are infinitely many $n$ where $K_{M}(A \uparrow h(n)) \geq n$.

Theorem 2.12 (Hölzl et al. [13]). A set is not totally i.o. complex iff it is Schnorr trivial.

\section{Characterization of Schnorr triviality via de- cidable prefix-free machines}

Schnorr randomness has a characterization via decidable prefix-free machines (Theorem 2.1). Then the following reducibility should have a strong relation to Schnorr randomness.

Definition 3.1. $A$ set $A$ is called weakly decidable prefix-free machine reducible to a set $B$ (denoted by $A \leq_{\mathrm{wdm}} B$ ) if for each decidable prefix-free machine $M$ and a computable order $g$ there exists a decidable prefix-free machine $N$ such that

$$
K_{N}\left(A\lceil n) \leq K_{M}(B \nmid n)+g(n)+O(1) .\right.
$$

First we see basic properties.

Proposition 3.2. The relation $\leq_{\mathrm{wdm}}$ is reflexive and transitive.

Proof. The reflexivity is immediate.

Let $A \leq_{\mathrm{wdm}} B$ and $B \leq_{\mathrm{wdm}} C$. Let $g$ be a computable order. For a decidable prefix-free machine $M$ and the order $g / 2$ there exists a decidable prefix-free machine $N$ such that $K_{N}(B \nmid n) \leq K_{M}(C\lceil n)+g(n) / 2+O(1)$. For this $N$ and the order $g / 2$ there exists a decidable prefix-free machine $L$ such that $K_{L}\left(A\lceil n) \leq K_{N}(B \nmid n)+g(n) / 2+O(1)\right.$. Hence $K_{L}\left(A\lceil n) \leq K_{M}(C \uparrow\right.$ $n)+g(n)+O(1)$. 
The following is also immediate.

Theorem 3.3. If a set $A$ is Schnorr random and $A \leq_{\mathrm{wdm}} B$, then $B$ is Schnorr random.

Proof. Let $M$ be a decidable prefix-free machine and $g$ be a computable order. Then there exists a decidable prefix-free machine $N$ such that $K_{N}(A\lceil n) \leq$ $K_{M}(B \nmid n)+g(n) / 2+O(1)$. Since $A$ is Schnorr random, $K_{N}(A \nmid n) \geq n-$ $g(n) / 2-O(1)$. Then it follows that $K_{M}(B\lceil n) \geq n-g(n)-O(1)$. Since $M$ and $g$ are arbitrary, $B$ is Schnorr random by Theorem 2.1.

We give a characterization of Schnorr triviality via the relation $\leq_{\mathrm{wdm}}$.

Theorem 3.4. $A$ set $A$ is Schnorr trivial iff $A \leq_{\mathrm{wdm}} \emptyset$.

This theorem follows from Theorem 3.5 but we give a direct short proof here.

Proof. ("if" direction) Suppose $A \leq_{\mathrm{wdm}} \emptyset$. Let $h$ be a computable order. Then there exists a computable order $g$ such that $g \circ h(n) \leq n / 2$. Note that $g \circ$ $h$ is computable order. Let $M$ be a decidable prefix-free machine such that $K_{M}(h(n)) \leq 2 \log n+O(1)$ for almost all $n$. Since $A \leq_{\mathrm{wdm}} \emptyset$, there exists a decidable prefix-free machine $N$ such that

$$
K_{N}\left(A\lceil h(n)) \leq K_{M}(h(n))+g(h(n))+O(1) \leq 2 \log n+n / 2+O(1)<n\right.
$$

for almost all $n$. By Theorem 2.12, $A$ is Schnorr trivial.

("only if" direction) Suppose that $A$ is Schnorr trivial. Then $A$ is computably tt-traceable by Theorem 2.10 .

We show $A \leq_{\text {wdm }} \emptyset$. Let $M$ be a decidable prefix-free machine and $g$ be an order. Let $L$ be a decidable prefix-free machine such that $K_{L}(n) \leq 2 \log n$ for almost all $n$ where we identify a natural number $n$ with the string $0^{n}$. Then

$$
\max \left\{K_{L}(i) \mid 1 \leq i \leq n\right\} \leq 2 \log n
$$

for almost all $n$. Let $f(n)=A\left\lceil n\right.$. Then $f \leq_{t t} A$. Hence there exists a computable trace $\left\{T_{n}\right\}$ such that $\left|T_{n}\right| \leq g(n)$ and $f(n) \in T_{n}$ for all $n$.

Let $N$ be a decidable prefix-free machine such that $N(\sigma \tau)$ is the $L(\tau)$-th element in $T_{M(\sigma)}$. Then

$K_{N}\left(A\lceil n)=K_{N}(f(n)) \leq K_{M}(n)+\max \left\{K_{L}(i) \mid 1 \leq i \leq g(n)\right\} \leq K_{M}(n)+g(n)\right.$

for almost all $n$. Then $A \leq_{\mathrm{wdm}} \emptyset$.

Actually, wdm-reducibility is equivalent to Schnorr reducibility.

Theorem 3.5. For all sets $A$ and $B$,

$$
A \leq_{\mathrm{Sch}} B \Longleftrightarrow A \leq_{\mathrm{wdm}} B .
$$


Let us recall the $\mathrm{KC}$ theorem [9, Theorem 3.6.1]. A KC set is a computable sequence of pairs $\left\{\left\langle d_{i}, \tau_{i}\right\rangle\right\}$ with $d_{i} \in \mathbb{N}$ and $\tau_{i} \in 2^{<\omega}$ such that $\sum_{i} 2^{-d_{i}} \leq 1$. Then there is a prefix-free machine $M$ and strings $\sigma_{i}$ of length $d_{i}$ such that $M\left(\sigma_{i}\right)=\tau_{i}$ for all $i$ and $\operatorname{dom}(M)=\left\{\sigma_{i}: i \in \mathbb{N}\right\}$. we call a pair $\langle d, \tau\rangle$ a request. The weight of a request $\langle d, \tau\rangle$ is $2^{-d}$. The weight of a computable sequence of requests $\left\{\left\langle d_{i}, \tau_{i}\right\rangle\right\}$ is the sum of the weights of the requests. Thus a $\mathrm{KC}$ set is a computable sequence of requests with the weight less than or equal to 1.

Lemma 3.6. For every decidable prefix-free machine $M$ and every computable order $g$, there exists a computable measure machine $N$ such that

$$
K_{N}(\sigma) \leq K_{M}(\sigma)+g(|\sigma|)+O(1) .
$$

Proof. Let $M$ be a decidable prefix-free machine and $g$ be a computable order. We define a $\mathrm{KC}$ set $S$ by

$$
S=\{\langle|\tau|+g(|\sigma|)+1, \sigma\rangle: M(\tau)=\sigma \text { and }|\tau| \leq 2|\sigma|+2\} .
$$

Clearly $S$ is a c.e. set. Furthermore

$$
\sum_{\langle\rho, \sigma\rangle \in S} 2^{-|\rho|} \leq \sum_{M(\tau)=\sigma} 2^{-|\tau|-g(|\sigma|)-1} \leq \sum_{M(\tau)=\sigma} 2^{-|\tau|-1} \leq 2^{-1} .
$$

We claim that the weight of the KC set is a computable real. For each $n \in \mathbb{N}$, define a uniformly c.e. set $S_{n}$ by

$$
S_{n}=\{\langle|\tau|+g(|\sigma|)+1, \sigma\rangle: M(\tau)=\sigma,|\tau| \leq 2|\sigma|+2 \text { and }|\tau|<2 n+2\} .
$$

Then $S_{n} \uparrow S$. Since $M$ is decidable and $S_{n}$ is a finite set, the weight of $S_{n}$ is computable for each $n$. If $\langle|\tau|+g(|\sigma|)+1, \sigma\rangle \in S \backslash S_{n}$, then $|\sigma| \geq n$. Hence

$$
\sum_{\langle\rho, \sigma\rangle \in S \backslash S_{n}} 2^{-|\rho|} \leq \sum_{M(\tau)=\sigma,|\sigma| \geq n} 2^{-|\tau|-g(|\sigma|)-1} \leq 2^{-g(n)-1}
$$

where the last inequality holds by prefix-freeness of $M$. It follows that the weight of $S$ is computable.

We define another $\mathrm{KC}$ set $T$ by

$$
T=\left\{\langle 2|\sigma|+2, \sigma\rangle: \sigma \in 2^{*}\right\} .
$$

Then $T$ is a c.e. set and the weight of $T$ is

$$
\sum_{\sigma \in 2^{*}} 2^{-2|\sigma|-2}=\sum_{n=0}^{\infty} \sum_{\sigma \in 2^{n}} 2^{-2|\sigma|-2}=\sum_{n=0}^{\infty} 2^{n} \cdot 2^{-2 n-2}=\sum_{n=0}^{\infty} 2^{-n-2}=2^{-1} .
$$

Hence $U=S \cup T$ is a $\mathrm{KC}$ set with a computable weight.

Let $N$ be a computable measure machine constructed from $U$ by the $\mathrm{KC}$ theorem. For each $\sigma$, let $\tau_{\sigma}$ be a string such that

$$
M\left(\tau_{\sigma}\right)=\sigma \text { and } K_{M}(\sigma)=\left|\tau_{\sigma}\right| .
$$


If $\left|\tau_{\sigma}\right| \leq 2|\sigma|+2$, then

$$
K_{N}(\sigma) \leq K_{M}(\sigma)+g(|\sigma|)+1
$$

If $\left|\tau_{\sigma}\right|>2|\sigma|+2$, then

$$
K_{N}(\sigma) \leq 2|\sigma|+2<K_{M}(\sigma) \leq K_{M}(\sigma)+g(|\sigma|)+1 .
$$

Thus the lemma is proved.

Proposition 3.7. Let $A, B$ be sets. If $A \leq_{\mathrm{Sch}} B$, then $A \leq_{\mathrm{wdm}} B$.

Proof. Suppose that $A \leq_{\text {Sch }} B$. Let $M$ be a decidable prefix-free machine $M$ and $g$ be a computable order.

By Lemma 3.6, there exists a computable measure machine $M^{\prime}$ such that

$$
K_{M^{\prime}}(B \nmid n) \leq K_{M}(B \nmid n)+g(n)+O(1) .
$$

Since $A \leq_{\mathrm{Sch}} B$, there exists a computable measure machine $N$ such that

$$
K_{N}\left(A\lceil n) \leq K_{M^{\prime}}(B \nmid n)+O(1) .\right.
$$

Then $N$ is a decidable prefix-free machine and, by (1) and (2), we have

$$
K_{N}\left(A\lceil n) \leq K_{M}(B \nmid n)+g(n)+O(1) .\right.
$$

Since $M$ and $g$ are arbitrary, we have $A \leq_{\mathrm{wdm}} B$.

Lemma 3.8. For every computable measure machine $M$, there exists a decidable prefix-free machine $N$ and a computable order $g$ such that

$$
K_{N}(\sigma) \leq K_{M}(\sigma)-g(|\sigma|)+O(1) .
$$

Proof. We can assume that $\operatorname{dom}(M)$ is not a finite set without loss of generality. We further assume that $|\sigma| \leq 2|\tau|+2$ for each $\sigma, \tau$ such that $M(\tau)=\sigma$.

For each $m$, we define a set $S_{m}$ by

$$
S_{m}=\{\tau: M(\tau) \downarrow \text { and }|\tau|<m\} .
$$

Then $S_{m} \uparrow \operatorname{dom}(M)$ as $m \rightarrow \infty$. It should be noted that $\llbracket \operatorname{dom}(M) \backslash S_{m} \rrbracket>0$ for all $m$ by the assumption. Then there exists a strictly increasing function $h: \mathbb{N} \rightarrow \mathbb{N}$ such that

$$
m>h(n) \Longrightarrow \llbracket \operatorname{dom}(M) \backslash S_{m} \rrbracket<2^{-n} .
$$

Since $M$ is a computable measure machine, $h$ can be computable. Then there exists a computable order $g$ such that

$$
2^{g(2 h(n+1)+1)} \leq n \text { for all } n \text { and } g(2 k+2) \leq k / 2 \text { for all } k .
$$


Let $C \in \omega$ be sufficiently large. We will be more precise later. We define a KC set $W$ as follows: if $M(\tau)=\sigma$, then we enumerate the pair $\langle|\tau|-g(|\sigma|)+C, \sigma\rangle$ into $W$. Then the weight of $W$ is

$$
\begin{aligned}
\sum_{M(\tau)=\sigma} 2^{-|\tau|+g(|\sigma|)-C} & =\sum_{n=0}^{\infty} \sum_{m=h(n)}^{h(n+1)-1} \sum_{M(\tau)=\sigma,|\tau|=m} 2^{-|\tau|+g(|\sigma|)-C} \\
& \leq \sum_{n=0}^{\infty} 2^{g(2 h(n+1)+1)-C} \llbracket \operatorname{dom}(M) \backslash S_{m} \rrbracket \\
& <\sum_{n=0}^{\infty} n \cdot 2^{-n-C} \leq 1
\end{aligned}
$$

for sufficiently large $C$.

Let $N$ be the prefix-free machine constructed from $W$ by the KC theorem. We claim that $N$ is decidable. Let $m \in \mathbb{N}$. Suppose that $M(\tau)=\sigma$ and $0 \leq|\tau|-g(|\sigma|)+C<m$. It should be noted that

$$
|\tau|-g(|\sigma|) \geq|\tau|-g(2|\tau|+2) \geq|\tau|-|\tau| / 2=|\tau| / 2 .
$$

Then $|\tau|<2(m-C)$. Hence, by enumerating $\tau$ such that $|\tau|<2(m-C)$, we can enumerate all $\rho$ such that $\rho \in \operatorname{dom}(N)$ and $|\rho|<m$.

For each $\sigma \in \operatorname{rng}(M)$, let $\tau_{\sigma}$ be such that $M\left(\tau_{\sigma}\right)=\sigma$ and $K_{M}(\sigma)=\left|\tau_{\sigma}\right|$. Then

$$
K_{N}(\sigma) \leq\left|\tau_{\sigma}\right|-g(|\sigma|)+C=K_{M}(\sigma)-g(|\sigma|)+C .
$$

Thus the lemma is proved.

Proposition 3.9. Let $A, B$ be sets. If $A \leq_{\mathrm{wdm}} B$, then $A \leq_{\mathrm{Sch}} B$.

Proof. Suppose that $A \leq_{\text {wdm }} B$. Let $M$ be a computable measure machine. By Lemma 3.8, there exists a decidable prefix-free machine $M^{\prime}$ and a computable order $g$ such that

$$
K_{M^{\prime}}(B \nmid n) \leq K_{M}(B \nmid n)-g(n)+O(1) .
$$

Since $A \leq_{\text {wdm }} B$, there exists a decidable prefix-free machine $N^{\prime}$ such that

$$
K_{N^{\prime}}\left(A\lceil n) \leq K_{M^{\prime}}(B \nmid n)+g(n) / 2+O(1) .\right.
$$

Again, by Lemma 3.6, there exists a computable measure machine $N$ such that

$$
K_{N}\left(A\lceil n) \leq K_{N^{\prime}}(A\lceil n)+g(n) / 2+O(1) .\right.
$$

By combining these, we have

$$
K_{N}\left(A\lceil n) \leq K_{M}(B \nmid n)+O(1) .\right.
$$

Thus the proposition is proved. 


\section{Preorderings related to uniform Schnorr ran- domness}

In this section we study a uniform-Schnorr-randomness version of the equivalence between LK-reducibility and LR-reducibility.

First recall the following definitions and facts. The reducibility $A \leq_{L K} B$ is defined by

$$
K^{B}(\sigma) \leq K^{A}(\sigma)+O(1) .
$$

The reducibility $A \leq_{L R} B$ is defined by that every Martin-Löf random set relative to $B$ is Martin-Löf random relative to $A$. Recall that a set is called low for $K$ if $A \leq_{L K} \emptyset$, and that a set is called low for Martin-Löf randomness if $A \leq_{L R} \emptyset$, and these notions are equivalent. Kjos-Hanssen et al. [14] strengthened this equivalence to the equivalence between two reducibility of $\leq_{L R}$ and $\leq_{L K}$.

By the results in Franklin and Stephan [10] and Miyabe [18], lowness for uniformly computable measure machines and lowness for uniform Schnorr randomness are euiqvalent. Then we strengthned this equivalence to the equivalence between reducibilities.

This section is dedicated to the proof of the following theorem.

Theorem 4.1. The followings are equivalent for $A, B \in 2^{\omega}$.

(i) Every Schnorr random set uniformly relative to $B$ is Schnorr random uniformly relative to $A$.

(ii) Every Schnorr test uniformly relative to A is covered by a Schnorr test uniformly relative to $B$.

(iii) For each uniformly computable measure machine $M$, there exists a uniformly computable measure machine $N$ such that

$$
K_{N^{B}}(\sigma) \leq K_{M^{A}}(\sigma)+O(1) .
$$

(iv) Each strictly bounded and uniformly Schnorr function $g: 2^{\omega} \rightarrow \tau$, there is a strictly bounded and uniformly Schnorr function $h: 2^{\omega} \rightarrow \tau$ such that $g(A) \subseteq h(B)$.

(v) For every computable function $f: 2^{\omega} \times \mathbb{N} \rightarrow \mathbb{R}^{+}$such that the function $X \mapsto \sum_{n=0}^{\infty} f(X, n)$ is computable, there exists a computable function $g$ : $2^{\omega} \times \mathbb{N} \rightarrow \mathbb{R}^{+}$such that the function $X \mapsto \sum_{n=0}^{\infty} g(X, n)$ is computable and $f(A, n) \leq g(B, n)$ for all $n$.

We show the equivalence among these by showing

$$
\text { (iii) } \Rightarrow \text { (ii) } \Rightarrow \text { (i) } \Rightarrow \text { (iv) } \Rightarrow \text { (v) } \Rightarrow \text { (iii). }
$$


Proof of (iii) $\Rightarrow($ ii). Let $f$ be a uniform Schnorr test. Then there exists a uniformly computable measure machine $M$ such that

$$
\bigcap_{n} f(A, n) \subseteq\left\{X:(\forall d)(\exists n) K_{M^{A}}(X\lceil n)<n-d\} .\right.
$$

By the hypothesis of (iii), there exists a uniformly computable measure machine $N$ such that

$$
K_{N^{B}}(\sigma) \leq K_{M^{A}}(\sigma)+O(1) .
$$

Then there exists a uniform Schnorr test $f^{\prime}$ such that

$$
\left\{X:(\forall d)(\exists n) K_{N^{B}}(X\lceil n)<n-d\} \subseteq \bigcap_{n} f^{\prime}(B, n) .\right.
$$

Thus the Schnorr null set $\bigcap_{n} f(A, n)$ uniformly relative to $A$ is covered by the Schnorr null set $\bigcap_{n} f^{\prime}(B, n)$ uniformly relative to $B$. Since $f$ is arbitrary, (iii) implies (ii).

Proof of $($ ii $) \Rightarrow(i)$. Suppose that $X$ is not Schnorr random uniformly relative to $A$. Then $X$ is covered by a Schnorr test uniformly relative to $A$, which is covered by a Schnorr test uniformly relative to $B$ by the hypothesis of (ii). Then $X$ is not Schnorr random uniformly relative to $B$.

Our remaining proofs mostly follow the argument in [2]

\subsection{Proof of $(\mathrm{i}) \Rightarrow$ (iv)}

First we recall some facts from Bienvenu and Miller [2].

An open set is called bounded if its measure is less than 1. A test is a non-increasing sequence $\left\{U_{n}\right\}$ of open sets such that $\bigcap_{n} U_{n}$ has measure 0 . For $U \subseteq 2^{\omega}$ and $\sigma \in 2^{<\omega}$, we set

$$
(U \mid \sigma)=\left\{X \in 2^{\omega}: \sigma X \in U\right\} .
$$

We say that a c.e. open set is a Schnorr set if its measure is computable.

The first fact to uniformly relativize here is the following.

Theorem 4.2 (Bienvenu and Miller [2]). The following are equivalent for a set $X \in 2^{\omega}$ :

(i) $X$ is not Schnorr random.

(ii) There is a bounded Schnorr open set $U$ such that all tails of $X$ belong to $U$.

(iii) $X \in U^{\omega}$ for some bounded Schnorr prefix-free subset $U$ of $2^{*}$.

The relativization of this theorem to $A$ gives the equivalence among the following. 
(i) $X$ is not Schnorr random relative to $A$.

(ii) There is a bounded open set $U$ such that $\mu(U)$ is computable from $A$ and all tails of $X$ belong to $U$.

(iii) $X \in U^{\omega}$ for some bounded prefix-free subset $U$ of $2^{<\omega}$ such that $\mu(U)$ is computable from $A$.

In contrast, the uniform relativization of this theorem to $A$ gives the following.

We say that a computable function $g: 2^{\omega} \rightarrow \tau$ is a uniformly Schnorr function if the function $X \mapsto \mu(g(X))$ is computable. A computable function $g: 2^{\omega} \rightarrow \tau$ is strictly bounded if $\sup _{X \in 2^{\omega}} \mu(g(X))<1$. Then the following are equivalent.

(i) $X$ is not Schnorr random uniformly relative to $A$.

(ii) There is a strictly bounded and uniformly Schnorr function $g$ such that all tails of $X$ belong to $g(A)$.

(iii) $X \in(g(A))^{\omega}$ for some c.e. function $g$ from $2^{\omega}$ to the subsets of $2^{*}$ such that $g(X)$ is prefix-free for all $X$ and the function $X \mapsto \llbracket g(X) \rrbracket$ is a strictly bounded and uniformly Schnorr function.

Here a function $g$ from $2^{\omega}$ to some discrete space is c.e. if and only if there is a computable function $h$ from $2^{\omega} \times \mathbb{N} g(X)=\sigma \downarrow \Longleftrightarrow h(X, s)=\sigma \downarrow$ for some $s$.

Lemma 4.3 (Bienvenu and Miller [2]). Let $\mathcal{C}$ be a class of bounded open subsets of $2^{\omega}$. Let $\left\{T_{n}^{e}\right\}_{e, n \in \mathbb{N}}$ be a countable family of tests (i.e., for all e, $\left\{T_{n}^{e}\right\}_{n \in \mathbb{N}}$ is a test) such that $\left\{T_{n}^{e}\right\}$ belongs to $\mathcal{C}$ for all e, $n$. Suppose we have the following closure properties.

(P1) For all $U \in \mathcal{C}$ and $\sigma \in 2^{<\omega}$, if $\mu(U \mid \sigma)<1$, then there exists a $V \in \mathcal{C}$ such that $(U \mid \sigma) \subseteq V$.

(P2) For all $U \in \mathcal{C}$, there exists $a V \in \mathcal{C}$ such that $U \subseteq V$, and for all $\sigma \in 2^{<\omega}$, if $\mu(U \mid \sigma)=1$, then $[\sigma] \subseteq V$.

(P3) For all $U \in \mathcal{C}$, and $\sigma \in 2^{<\omega}$, if $\mu(U \mid \sigma)<1$, then for all $e \in \mathbb{N}$, there exists $n_{e} \in \mathbb{N}$ and $V \in \mathcal{C}$ such that $\left(U \cup T_{n_{e}}^{e}\right) \subseteq V$ and $\mu(V \mid \sigma)<1$.

Finally, let $W$ be a prefix-free subset of $2^{<\omega}$ such that $[W]$ cannot be covered by any open set $U \in \mathcal{C}$. Then there exists $X \in W^{\omega}$ that passes all tests $T^{e}$.

Bienvenu and Miller [2] uses the following proposition to study lowness for Schnorr randomness.

Proposition 4.4 (Bienvenu and Miller [2]). Let $\mathcal{C}$ be the class of bounded Schnorr open sets and $T^{e}$ be the family of Schnorr tests. Then the hypotheses (P1,P2,P3) of Lemma 4.3 are satisfied for these classes. 
The uniform relativization gives the following.

Proposition 4.5. Let $A$ be a set. Let $\mathcal{C}$ be the class of open sets $g(A)$ such that $g: 2^{\omega} \rightarrow \tau$ is a strictly bounded and uniformly Schnorr function. Let $T^{e}$ be the family of Schnorr tests uniformly relative to A. Then the hypotheses (P1,P2,P3) of Lemma 4.3 are satisfied for these classes.

Proof of $(i) \Rightarrow$ (iv) of Theorem 4.1. Suppose that there exists a strictly bounded and uniformly Schnorr function $g: 2^{\omega} \rightarrow \tau$ such that $g(A) \not \subset h(B)$ for all strictly bounded and uniformly Schnorr function $h$. Let $W$ be a prefix-free subset of $2^{<\omega}$ such that $[W]=g(A)$. Apply Lemma 4.3 for this $W$. Then there exists $X \in W^{\omega}$ that passes all Schnorr tests uniformly relative to $B$. Then $X$ is Schnorr random uniformly relative to $B$ and is not Schnorr random uniformly relative to $A$ by the uniform relativization of Theorem 4.2.

\subsection{Proof of (iv) $\Rightarrow(\mathrm{v})$}

We use the same techniques as the proof of Proposition 5.1 in the revised version of [2] in arXiv.

Proof of $(i v) \Rightarrow(v)$ of Theorem 4.1. By the usual identification of $\omega$ to $\omega \times \omega$ and of $2^{\omega}$ to $[0,1]$, we identify $2^{\omega}$ with $[0,1]^{\omega}$. For all $n \in \omega$ and $\alpha \in[0,1]$, set

$$
\mathcal{B}_{n, \alpha}=\left\{X \in[0,1]^{\omega}: X_{n} \in[0, \alpha)\right\} .
$$

Let $f: 2^{\omega} \times \omega \rightarrow \mathbb{R}^{+}$be a computable function such that $X \mapsto \sum_{n=0}^{\infty} f(X, n)$ is computable. Since $2^{\omega}$ is compact, $\sup _{X \in 2^{\omega}} \sum_{n=0}^{\infty} f(X, n)$ is finite. Without loss of generality, we can assume that $\sum_{n=0}^{\infty} f(X, n) \leq 1$. Consider the computable function $h: 2^{\omega} \rightarrow \tau$ defined by

$$
h(X)=\bigcup_{n} \mathcal{B}_{n, f(X, n)} .
$$

Then

$$
\mu(h(X))=1-\prod_{n}\left(1-\mu\left(\mathcal{B}_{n, f(X, n)}\right)\right)=1-\prod_{n}(1-f(X, n)) .
$$

This can be reformulated as

$$
\log (1-\mu(g(X)))=\sum_{n} \log (1-f(X, n)) .
$$

Since $f(X, n)$ tends to $0, X \mapsto \sum_{n} \log (1-f(X, n))$ is computable. Thus, $g$ is a strictly bounded and uniformly Schnorr function. By hypothesis (iv), there is a strictly bounded and uniformly Schnorr function $k: 2^{\omega} \rightarrow \tau$ such that $h(A) \subseteq k(B)$.

Let $\delta>0$ be such that $\sup _{X \in 2^{\omega}} \mu(k(X))<1-\delta$. For all $n \in \omega$, let $k(X, n)$ be the approximation of $k(X)$ with precision $2^{-n-c}$ where $c \in \omega$ to be 
specified shortly. That is, $k(X, n)$ is a clopen set for which an exact index can be uniformly computed from $X$ and $n$, and $\mu(k(X) \backslash k(X, n))<2^{-n-c}$. Now define the function $g: 2^{\omega} \times \omega \rightarrow \mathbb{R}$ by

$$
g(X, n)=\max \left\{\alpha \in[0,1]: \mu\left(\mathcal{B}_{n, \alpha} \backslash k(X, n)\right) \leq 2^{-n-c}\right\}
$$

Note that $g$ is computable. Also note that $g(B, n) \geq f(A, n)$ because $\mathcal{B}_{n, f(A, n)} \subseteq$ $h(A) \subseteq k(B)$ and

$$
\mu\left(\mathcal{B}_{n, f(A, n)} \backslash k(B, n)\right) \leq \mu(k(B) \backslash k(B, n)) \leq 2^{-n-c} .
$$

Now it suffices to show that the function $X \mapsto \sum_{n} g(X, n)$ is computable. Let $m$ be a fixed integer. Since $k(X, m)$ is a clopen set, one can effectively find an integer $N=N(k)$ such that $k(X, m)$ is independent from $\left\{\mathcal{B}_{n, g(n)}: n \geq N\right\}$. By this independence, we have

$$
\begin{aligned}
\mu\left(\bigcup_{n>N} \mathcal{B}_{n, g(X, n)} \backslash k(X, m)\right) & =(1-\mu(k(X, m))) \mu\left(\bigcup_{n>N} \mathcal{B}_{n, g(X, n)}\right) \\
& >\delta \cdot \mu\left(\bigcup_{n>N} \mathcal{B}_{n, g(X, n)}\right) .
\end{aligned}
$$

On the other hand, we have

$$
\begin{aligned}
\mu\left(\bigcup_{n>N} \mathcal{B}_{n, g(X, n)} \backslash k(X)\right) & \leq \sum_{n>N} \mu\left(\mathcal{B}_{n, g(X, n)} \backslash k(X)\right) \\
& \leq \sum_{n>N} \mu\left(\mathcal{B}_{n, g(X, n)} \backslash k(X, n)\right) \\
& \leq \sum_{n>N} 2^{-n-c}=2^{-N-c} .
\end{aligned}
$$

By combining (3) and (4) and the fact that $\mu(k(X) \backslash k(X, m))<2^{-m-c}$, we obtain

$$
1-\prod_{n>N}(1-g(X, n))=\mu\left(\bigcup_{n>N} \mathcal{B}_{n, g(X, n)}\right) \leq \frac{2^{-N-c}+2^{-m-c}}{\delta} .
$$

We assume that $2^{-c+1}<\delta$ and $N \geq m$. Then

$$
1-\prod_{n>N}(1-g(X, n))<2^{-m}
$$

(By considering the case that $m=0$ and $N=0$, we have $1-\prod_{n}(1-g(X, n))<1$, which implies $\sum_{n} g(X, n)$ is finite.) Composing with $-\log$ on both sides, we obtain

$$
\sum_{n>N} g(X, n)<-\log \left(1-2^{-m}\right)=2^{-m+o(m)} .
$$

Hence $\sum_{n} g(X, n)$ is computable from $X$. 


\subsection{Proof of (v) $\Rightarrow$ (iii)}

We follow the proof of Proposition 27 in [2].

Proof of $(i v) \Rightarrow$ (iii) of Theorem 4.1. Let $M$ be a uniformly computable measure machine. The goal is to show the existence of a uniformly computable measure machine $N$ such that $K_{N^{B}}(\sigma) \leq K_{M^{A}}(\sigma)+O(1)$. Let $f: 2^{\omega} \times 2^{<\omega} \rightarrow \mathbb{R}^{+}$be the function defined by

$$
f(X, \sigma)=2^{-K_{M} X(\sigma)} .
$$

Then $f$ is lower semicomputable. Since the function $X \mapsto \sum_{\sigma \in \operatorname{dom}\left(M^{X}\right)} 2^{-|\sigma|}$ is computable, the function $X \mapsto \sum_{\sigma \in 2<\omega} f(X, \sigma)$ is computable. By the assumption of (iv), there is a computable function $g: 2^{\omega} \times 2^{<\omega} \rightarrow \mathbb{R}^{+}$such that $X \mapsto \sum_{\sigma \in 2<\omega} g(X, \sigma)$ is computable and $f(A, \sigma) \leq g(B, \sigma)$ for all $n$.

Since $2^{\omega}$ is compact, $\sup _{X \in 2^{\omega}} \sum_{\sigma} g(X, \sigma)$ is finite. Let $c \in \omega$ be a constant such that $\sum_{\sigma} g(X, \sigma) \leq 2^{c}$ for all $X \in 2^{\omega}$. Let

$$
L^{X}=\left\{\langle k, \sigma\rangle: g(X, \sigma) \geq 2^{-k+c+1}\right\} .
$$

Then

$$
\sum_{\langle k, \sigma\rangle \in L^{X}} 2^{-k}=\sum_{\sigma} 2^{-\lceil\log g(X, \sigma)-c-1\rceil+1} \leq 2^{-c} \sum_{\sigma} g(X, \sigma) \leq 1 .
$$

Thus, $L$ is a KC-set. Apply the $\mathrm{KC}$ theorem to construct a oracle prefix-free machine $N$ whose domain is a prefix-free set $\left\{p_{k, \sigma}^{X}:\langle k, \sigma\rangle \in L^{X}\right\}$ with $\left|p_{k, \sigma}^{X}\right|=$ $k$ and $N^{X}\left(p_{k, \sigma}^{X}\right)=\sigma$. Since the function $X \mapsto \sum_{\sigma} g(X, \sigma)$ is computable, the function $X \mapsto \sum_{\sigma} 2^{-\lceil\log g(X, \sigma)-c-1\rceil+1}$ is computable. Finally notice that

$$
K_{N^{B}}(\sigma) \leq-\log g(B, \sigma)+c+1 \leq-\log f(A, \sigma)+c+1 \leq K_{M^{A}}(\sigma)+c+1 .
$$

\section{Base for uniform Schnorr tests}

We say that a set $A$ is a base for ML-randomness if $A \leq_{\mathrm{T}} X$ for some set $X$ that is ML-random relative to A. It is known that being a base for MLrandomness is equivalent to $K$-triviality. Then we would like to have a basetype characterization of Schnorr triviality. One candidate is like this: a set $A$ such that $A \leq_{\mathrm{tt}} X$ for some set $X$ that is uniform Schnorr random relative to $A$. However this notion is not equivalent to Schnorr triviality. Actually Franklin and Stephan [10] showed that there exists a Schnorr trivial set that is not truth-table reducible to any Schnorr random set.

It should be noted that the following notions are equivalent:

(i) Schnorr triviality (Definition 2.7),

(ii) computable tt-traceability (Definition 2.8), 
(iii) non-totally i.o. complexity (Definition 2.11).

Notice that all of them have the following form: a set $A$ is Schnorr trivial iff for any computable object, there exists another computable object such that $A$ is in some object. Then we define a base for uniform Schnorr tests in a similar manner and show the equivalence to Schnorr triviality.

Definition 5.1. Let $d$ be a computable martingale uniformly relative to $A$. A set $X$ is computably random uniformly relative to $A$ for $d$ if $d(X\lceil n) \leq O(1)$. A set $X$ is Schnorr random uniformly relative to $A$ for $d$ if $d(X\lceil n) \leq h(n)+O(1)$ for each computable order $h$.

Definition 5.2. A set $A$ is a base for uniformly computable martingales if, for each computable martingale $d$ uniformly relative to $A$, there exists a set $B$ such that $A \leq_{\mathrm{tt}} B$ and $B$ is computably random uniformly relative to $A$ for $d$.

$A$ set $A$ is a base for uniform Schnorr tests if, for each computable martingale $d$ uniformly relative to $A$, there exists a set $B$ such that $A \leq_{\mathrm{tt}} B$ and $B$ is Schnorr random uniformly relative to $A$ for $d$.

Remark 5.3. The definition of a base for uniform Schnorr tests uses a uniformly computable martingale rather than a uniform Schnorr test. The terminology makes sense because of the correspondence of tests and martingales.

Then we show that being a base for uniform Schnorr tests is equivalent to Schnorr triviality. Further we show that being a base for tt-reducible martingales is also equivalent.

Theorem 5.4. The following are equivalent for a set A:

(i) A is Schnorr trivial,

(ii) $A$ is a base for uniformly computable martingales,

(iii) $A$ is a base for uniform Schnorr tests.

First note that (ii) $\Rightarrow$ (iii) is immediate. We prove the remaining implications by showing Lemma 5.5 and Lemma 5.6.

Lemma 5.5. If a set is a base for uniform Schnorr tests, then it is Schnorr trivial.

Franklin and Stephan [10] stated as a proposition that if $A \leq_{\mathrm{tt}} B$ and $B$ is Schnorr random uniformly relative to $A$, then $A$ is Schnorr trivial. The proof actually showed something stronger: for any $A, B \in 2^{\omega}$ such that $A \leq_{\mathrm{tt}} B$, at least one of the following holds:

(i) There exists a computable martingale $d$ uniformly relative to $A$ such that $B$ is not Schnorr random uniformly relative to $A$ for $d$.

(ii) $A$ is Schnorr trivial.

Hence, if $A$ is not Schnorr trivial, then $A$ is not a base for uniform Schnorr tests. 
Lemma 5.6. If a set is Schnorr trivial, then it is a base for uniformly computable martingales.

For the proof, we use the Space Lemma.

Lemma 5.7 (Space Lemma; see Merkle and Mihailović [17]). Given a rational $\delta>1$ and integer $k>0$, we can compute a length $l(\delta, k)$ such that, for any martingale $d$ and any $\sigma$,

$$
\left|\left\{\tau \in 2^{l(\delta, k)} \mid d(\sigma \tau) \leq \delta d(\sigma)\right\}\right| \geq k .
$$

Proof of Lemma 5.6. Let $A$ be a Schnorr trivial set and $d$ be a uniformly computable martingale. We assume that $d$ is $\mathbb{Q}_{2}$-valued without loss of generality. We will construct a set $B$ such that $A \leq_{\mathrm{tt}} B$ and $B$ is computably random uniformly relative to $A$ for $d^{A}$.

Let $r_{1}>r_{2}>\cdots>1$ be a computable sequence of rationals such that, letting $\beta_{i}=\prod_{j \leq i} r_{i}$, the sequence $\left\{\beta_{i}\right\}$ converges to some value $\beta$. Let $l_{s}=$ $l\left(r_{s}, s\right)$ be as in the Space Lemma and $\hat{l}_{s}=\sum_{i=1}^{s} l_{i}$.

Let $u$ be the use function of $d^{A}$. Then $n \mapsto \max \left\{u(\sigma) \mid \sigma \in 2^{n}\right\}$ is dominated by a computable function $r(n)$. We assume $u(n) \leq r(n)$ for all $n$. Since $A$ is computably tt-traceable, there exists a trace $\left\{T_{n}\right\}$ such that $\left|T_{n}\right| \leq n$ and $A\left\lceil r\left(\hat{l}_{n}\right) \in T_{n}\right.$ for all $n$. We further assume that any two elements in $T_{n}$ are distinct.

We construct $B=\lim _{s} \sigma_{s}$ as follows. Let $\sigma_{0}$ be the empty string. Then $d^{A}\left(\sigma_{0}\right) \leq 1$. Note that $\sigma_{0}=B \nmid 0$. For $s \geq 1$, we assume by induction that $d^{A}\left(\sigma_{s-1}\right) \leq \beta_{s-1}$ where $\sigma_{s-1}=B \nmid \hat{l}_{s-1}$. Then there are at least $s$ strings $\tau$ such that $d^{A}\left(\sigma_{s-1} \tau\right) \leq \beta_{s}$. Let $\tau_{m}$ be the lexicographically $m$-th least string among such strings. Let $\sigma_{s+1}=\sigma_{s-1} \tau_{m}$ when $A \uparrow r\left(\hat{l}_{s}\right)$ is the $m$-th element in $T_{s}$. Then $d^{A}\left(B \vdash \hat{l}_{s}\right)<\beta$ for all $s$. It follows that $B$ is computably random uniformly relative to $A$ for $d^{A}$.

Next we construct a reduction $\Phi$ to $X$ such that $A=\Phi^{B}$. We assume by induction that we have already known $\Phi^{X}\left\lceil r\left(\hat{l}_{s}\right)\right.$ for $s \geq 1$ where $\Phi^{X}\left\lceil r\left(\hat{l}_{1}\right)=\right.$ $A\left\lceil r\left(\hat{l}_{1}\right)\right.$. Since $r$ dominates the use function $u$ of $d$, we have $u\left(\hat{l}_{s}\right) \leq r\left(\hat{l}_{s}\right)$. Hence we can compute $d^{\Phi^{X}}\left(\left(X \uparrow \hat{l}_{s-1}\right) \tau\right)$ for all $\tau$ such that $|\tau|=l_{s}$. Decide whether a string $\tau$ satisfy $|\tau|=l_{s}$ and $d^{\Phi^{X}}\left(\left(X \vdash \hat{l}_{s-1}\right) \tau\right) \leq \beta_{s}$. Let $\tau_{m}$ be the lexicographically $m$-th least string among such strings. Search $m$ satisfying $X\left\lceil\hat{l}_{s}=\left(X\left\lceil\hat{l}_{s-1}\right) \tau_{m}\right.\right.$. If such an $m$ is found, then let $\Phi^{X}\left\lceil r\left(\hat{l}_{s+1}\right)\right.$ be the $m$-th element in $T_{s+1}$. Otherwise let $\Phi^{X}=\left(\Phi^{X}\left\lceil r\left(\hat{l}_{s}\right)\right) 0^{\omega}\right.$. Then $\Phi$ is a tt-reduction.

Finally we show $A=\Phi^{B}$. We prove $\Phi^{B}\left\lceil r\left(\hat{l}_{s+1}\right)=A\left\lceil r\left(\hat{l}_{s+1}\right)\right.\right.$ by induction on $s$. For $s=0$, this is true. Suppose this is true for $s-1$. Let $\tau_{m}$ be the lexicographically $m$-th least string $\tau$ such that $d^{A}\left(\sigma_{s-1} \tau\right) \leq \beta_{s}$. By the inductive hypothesis, $\Phi^{B}\left\lceil r\left(\hat{l}_{s}\right)=A\left\lceil r\left(\hat{l}_{s}\right)\right.\right.$. Then

$$
d^{\Phi^{B}}\left(\left(B \nmid \hat{l}_{s-1}\right) \tau\right) \leq \beta_{s} \Longleftrightarrow d^{A}\left(\sigma_{s-1} \tau\right) \leq \beta_{s} .
$$

Hence there exists only one $m_{0}$ such that $B \uparrow \hat{l}_{s}=\left(B \uparrow \hat{l}_{s-1}\right) \tau_{m_{0}}$ and $A \uparrow$ $r\left(\hat{l}_{s+1}\right)=\Phi^{B}\left\lceil r\left(\hat{l}_{s+1}\right)\right.$ is the $m_{0}$-th element in $T_{s+1}$. Then the equation is true for $s$. 


\section{Acknowledgement}

The author thanks Takayuki Kihara triggering this study and Laurent Bienvenu for crucial discussion on the proof of (iv) $\Rightarrow(\mathrm{v})$ of Theorem 4.1. This work was partly supported by GCOE, Kyoto University and JSPS KAKENHI 23740072.

\section{References}

[1] L. Bienvenu and W. Merkle. Reconciling data compression and kolmogorov complexity. In L. Arge, C. Cachin, T. Jurdziński, and A. Tarlecki, editors, Automata, Languages and Programming, volume 4596 of Lecture Notes in Computer Science, pages 643-654, Berlin, 2007. Springer.

[2] L. Bienvenu and J. S. Miller. Randomness and lowness notions via open covers. Annals of Pure and Applied Logic, 163:506-518, 2012. arXiv:1303.4902.

[3] V. Brattka. Computability over topological structures. In S. B. Cooper and S. S. Goncharov, editors, Computability and Models, pages 93-136. Kluwer Academic Publishers, New York, 2003.

[4] V. Brattka, P. Hertling, and K. Weihrauch. A tutorial on computable analysis. New Computational Paradigms, pages 425-491, 2008.

[5] D. Diamondstone, N. Greenberg, and D. Turetsky. A van lambalgen theorem for demuth randomness. Submitted.

[6] R. Downey, N. Greenberg, N. Mihailovic, and A. Nies. Lowness for computable machines. In C. T. Chong, Q. Feng, T. A. Slaman, W. H. Woodin, and Y. Yang, editors, Computational Prospects of Infinity: Part II, Lecture Notes Series, pages 79-86. World Scientific Publishing Company, 2008.

[7] R. Downey and E. Griffiths. Schnorr randomness. Journal of Symbolic Logic, 69(2):533-554, 2004.

[8] R. Downey, E. Griffiths, and G. LaForte. On Schnorr and computable randomness, martingales, and machines. Mathematical Logic Quarterly, 50(6):613-627, 2004.

[9] R. Downey and D. R. Hirschfeldt. Algorithmic Randomness and Complexity. Springer, Berlin, 2010.

[10] J. N. Y. Franklin and F. Stephan. Schnorr trivial sets and truth-table reducibility. Journal of Symbolic Logic, 75(2):501-521, 2010.

[11] J. N. Y. Franklin, F. Stephan, and L. Yu. Relativizations of randomness and genericity notions. Bulletin of the London Mathematical Society, 43(4):721733, 2011.

[12] D. Hirschfeldt, A. Nies, and F. Stephan. Using random sets as oracles. Journal of the London Mathematical Society, 75:610-622, 2007. 
[13] R. Hölzl and W. Merkle. Traceable sets. Theoretical Computer Science, pages $301-315,2010$.

[14] B. Kjos-Hanssen, J. S. Miller, and D. R. Solomon. Lowness notions, measure, and domination. Journal of the London Mathematical Society, 85(3):869-888, 2012.

[15] B. Kjos-Hanssen, A. Nies, and F. Stephan. Lowness for the class of Schnorr random reals. SIAM Journal on Computing, 35(3):647-657, 2005.

[16] P. Martin-Löf. The Definition of Random Sequences. Information and Control, 9(6):602-619, 1966.

[17] W. Merkle and N. Mihailović. On the construction of effective random sets. Mathematical Foundations of Computer Science, pages 568-580, 2002.

[18] K. Miyabe. Truth-table Schnorr randomness and truth-table reducible randomness. Mathematical Logic Quarterly, 57(3):323-338, 2011.

[19] K. Miyabe and J. Rute. Van Lambalgen's Theorem for uniformly relative Schnorr and computable randomness. To appear in Proceedings of the Twelfth Asian Logic Conference.

[20] A. Nies. Lowness properties and randomness. Advances in Mathematics, 197:274-305, 2005.

[21] A. Nies. Computability and Randomness. Oxford University Press, USA, 2009.

[22] C. P. Schnorr. Zufälligkeit und Wahrscheinlichkeit, volume 218 of Lecture Notes in Mathematics. Springer-Verlag, Berlin-New York, 1971.

[23] S. A. Terwijn and D. Zambella. Computational randomness and lowness. Journal of Symbolic Logic, 66(3):1199-1205, 2001.

[24] K. Weihrauch. Computable Analysis: an introduction. Springer, Berlin, 2000.

[25] K. Weihrauch and T. Grubba. Elementary Computable Topology. Journal of Universal Computer Science, 15(6):1381-1422, 2009. 